\title{
Cysteine 95 and other residues influence the regulatory effects of Histidine 69 mutations on Human Immunodeficiency Virus Type 1 protease autoprocessing
}

\author{
Liangqun Huang, Alyssa Hall, Chaoping Chen*
}

\begin{abstract}
Background: Regulated autoprocessing of HIV Gag-Pol precursor is required for the production of mature and fully active protease. We previously reported that H69E mutation in a pseudo wild type protease sequence significantly (>20-fold) impedes protease maturation in an in vitro autoprocessing assay and in transfected mammalian cells.

Results: Interestingly, H69E mutation in the context of a laboratory adapted NL4-3 protease showed only moderate inhibition ( 4-fold) on protease maturation. There are six point mutations (Q7K, L33I, N37S, L63I, C67A, and (95A) between the NL4-3 and the pseudo wild type proteases suggesting that the H69E effect is influenced by other residues. Mutagenesis analyses identified C95 as the primary determinant that dampened the inhibitory effect of H69E. L63 and C67 also demonstrated rescue effect to a less extent. However, the rescue was completely abolished when $\mathrm{H} 69$ was replaced by aspartic acid in the NL4-3 backbone. Charge substitutions of surface residues (E21, D30, E34, E35, and F99) to neutral or positively charged amino acids failed to restore protease autoprocessing in the context of H69E mutation.
\end{abstract}

Conclusions: Taken together, we suggest that residue 69 along with other amino acids such as C95 plus L63 and C67 to a less extent modulate precursor structures for the regulation of protease autoprocessing in the infected cell.

\section{Background}

Human immunodeficiency virus 1 (HIV-1) is a member of the lentivirus genus in the retroviradae superfamily. In the HIV infected cell, the unspliced genomic RNA also serves as mRNA for translation of two polyproteins: Gag and Gag-Pol [1,2]. Gag polyprotein is the primary viral determinant responsible for the assembly and release of progeny virions [3,4]. Gag-Pol polyprotein is produced as a result of regulated frameshifting that reads through the stop codon in the Gag reading frame $[5,6]$. In the Gag-Pol precursor, HIV protease is flanked $\mathrm{N}$-terminally by the transframe region (TFR) (Figure $1 \mathrm{~A})$ and C-terminally by the reverse transcriptase $[5,7]$. The embedded precursor protease has an intrinsic

\footnotetext{
* Correspondence: chaoping@colostate.edu

* Correspondence: chaoping@colostate.edu
Department of Biochemistry and Molecular Biology, Colorado State University, Fort Collins, Colorado, USA
}

(c) 2010 Huang et al; licensee BioMed Central Ltd. This is an Open Access article distributed under the terms of the Creative Commons Attribution License (http://creativecommons.org/licenses/by/2.0), which permits unrestricted use, distribution, and reproduction in any medium, provided the original work is properly cited.

ability to catalyze cleavages of a few sites in Gag and Gag-Pol polyproteins [8-10], but the full proteolytic activity is only associated with the mature protease after it is liberated from the precursor as a result of autoprocessing. The N-terminal cleavage is critical for protease maturation $[5,11]$ since blocking the $\mathrm{N}$-terminal cleavage abolishes the production of mature protease $[10,12]$. In contrast, mutations blocking the C-terminal cleavage have no significant influence on protease activity $[13,14]$.

The mature protease recognizes and cleaves at least 10 different sites in Gag and Gag-Pol polyproteins $[15,16]$. These sites are processed at rates that vary up to 400 fold in vitro $[17,18]$, probably due to the diversity of target sequences [19]. Among the five canonical HIV-1 Gag processing sites, the $\mathrm{p} 2 / \mathrm{NC}$ site appears to be the preferred substrate as both protease precursor and mature protease can cleave this site with high efficiency $[9,20]$. In contrast, mature protease is required for the 
cleavage at the $\mathrm{CA} / \mathrm{p} 2$ site $[17,21]$. Accurate and precise protease processing is absolutely required for the production of infectious progeny virions. Mutations that alter the time of processing or the order in which these sites are cleaved, or that produce incorrect cleavage at individual sites, cause the release of aberrant virions that are significantly less infectious [22-25].

The mature HIV protease is composed of 99 amino acids and is a member of the aspartyl protease family $[7,26,27]$. Unlike the cellular aspartic proteases that are active monomers, mature HIV protease exists as stable dimers $\left(K_{\mathrm{d}}<5 \mathrm{nM}\right)$ with the catalytic site formed at the dimer interface by two aspartic acids; each is contributed by one monomer [5]. Mutations that alter the aspartic acid to either asparagine or alanine abolish protease activity in vitro and in vivo [27-30]. In contrast to mature proteases that are stable dimers, protease precursors containing the $\mathrm{N}$-terminal TFR have a much higher dimer dissociation constant $\left(K_{\mathrm{d}}>500 \mu \mathrm{M}\right)$ and exhibit very low catalytic activity $[5,11]$. Transient protease precursor dimerization coupled with the N-terminal cleavage is concomitant with the formation of stable dimers and the appearance of full catalytic activity when purified protease precursors are refolded in vitro $[31,32]$ - a process defined as autocatalytic maturation or autoprocessing [5].

A pseudo wild type protease, which bears six point mutations (Q7K, L33I, N37S, L63I, C67A, and C95A) compared to the NL4-3 protease, has been previously optimized for NMR and kinetic studies of protease maturation [11]. Mutations Q7K, L33I, L63I minimize autoproteolysis; C67A and C95A prevent cysteine-thiol oxidation. We previously described that alteration of His 69 , a surface residue of the mature protease, to glutamic acid in the pseudo wild type protease sequence significantly blocks precursor autoprocessing both in $E$. coli and in transfected mammalian cells [33]. Biochemical analyses indicate that the mature H69E protease displayed a slightly lower catalytic activity comparable to the wild type protease. However, in vitro autoprocessing of $\mathrm{H} 69 \mathrm{E}$ precursor is drastically delayed, suggesting that H69E mutation may interfere with productive folding of the precursor. Interestingly, H69E mutation in the context of NL4-3 derived protease only demonstrated a moderate inhibitory effect on protease maturation. We sought here to define residues that contribute to the differential impacts on precursor autoprocessing. This information would provide insights into the molecular mechanism that regulates protease autoprocessing.

\section{Results}

\section{H69E mutation displayed different effects under two} different contexts

In our previous report, $\mathrm{H} 69 \mathrm{E}$ and other mutations were constructed in the context of a pseudo wild type ( $\mathrm{wt}^{\text {pse }}$ ) protease sequence, in which H69E significantly impedes precursor autoprocessing. Compared to the laboratory adapted NL4-3 derived protease, the pseudo wild type protease contains six point mutations (Figure 1A), but otherwise displays enzymatic kinetics similar to the wild type protease [34]. Mutations Q7K, L33I, and L63I are known to minimize autoproteolysis; and C67A/C95A mutations prevent aggregation of $E$. coli expressed protease mediated by cysteine thiol oxidation. To further understand the inhibition mechanism of H69E on protease autoprocessing, we first sought to examine the effects of H69E in the context of NL4-3 protease.

The previously described pNL-PR proviral construct was used to engineer the indicated mutations (Figure $1 \mathrm{~B})$, and the resulting plasmids were transfected into
A

\begin{tabular}{|l|l|||l|}
\hline MA & CA & NC \\
\hline
\end{tabular}

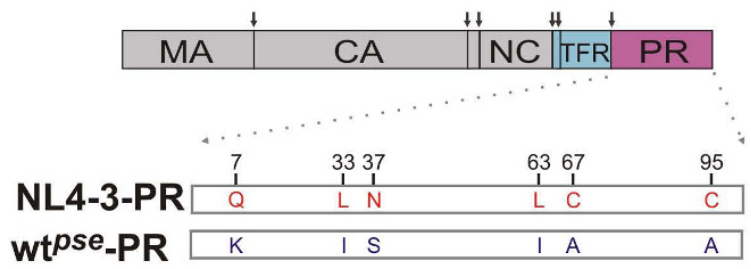

B

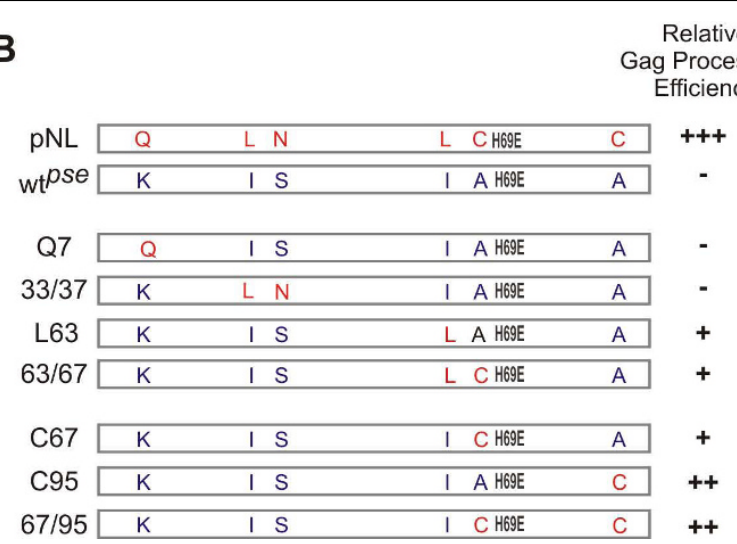

Figure 1 Schematic illustration of constructs with or without H69E mutation. (A) Organization of structural domains in the Gag and GagPR polyproteins: MA, matrix; CA, capsid (p24); NC, nucleocapsid; p6, late domain protein; TFR, transframe region; PR, protease. Straight arrows indicate the protease cleavage sites. Amino acids that are different between NL4-3 and we $t^{\text {pse }}$ proteases are denoted. (B) Schematic summary on H69E containing mutants and their relative Gag processing efficiencies. 
HEK 293 T cells for the study. Approximately equal amounts of total Gag proteins were detected in cell lysates suggesting similar expression efficiencies mediated by the pNL-PR proviruses. Also, the amounts of virus-like particle (VLP) released into the culture medium were similar to each other, indicating these mutations have minimal impact on virion production. In the absence of any protease activity, as with D25N mutant, the full length Gag polyprotein (p55) is the predominant product in transfected cells and the released VLPs (Figure 2 lane 10). In the presence of mature proteases as a result of effective autoprocessing, p24 was detected as the predominant band with little p25 and p55 (Figure 2 lanes 8 and 9). Consistent with our previous report, VLPs produced by wt ${ }^{p s e}$ H69E contained predominantly the full length Gag polyprotein and no processed p24, indicating lack of mature protease activity (Figure 2, lane 3). Interestingly, VLPs as well as cell lysates made by NL4-3 H69E showed some p24 proteins, suggesting an association of mature protease activity in both. We quantified the ratio of p24 to total p24-containing proteins as a measure of relative Gag processing efficiency to indirectly reflect autoprocessing activity, and our data demonstrated that $\mathrm{wt}^{\text {pse }}{ }^{\mathrm{H}} \mathrm{H} 69 \mathrm{E}$ mutation had $<5 \%$ of the wild type processing activity, i.e. > 20-fold inhibition; while NL4-3 H69E showed $\sim 25 \%$ of the wild type processing efficiency, i.e. $\sim 4$-fold inhibition (Figure $2 B$ ). Given that there are six point mutations between NL4-3 and $\mathrm{wt}^{p s e}$ protease, our data suggested that the inhibitory effect of H69E on protease autoprocessing is influenced by other residues.

\section{C95 and other residues dampened the inhibitory effect of H69E on protease autoprocessing}

In order to define residues that rescued protease autoprocessing in the NL4-3 H69E construct, we engineered a panel of $\mathrm{H} 69 \mathrm{E}$ proviruses replacing the six point mutations in the $\mathrm{wt}^{\text {pse }}$ backbone with the corresponding NL4-3 amino acids individually or in combination (Figure 1B) and tested their Gag processing efficiencies to evaluate autoprocessing activities (Figure 2). The $\mathrm{wt}^{\text {pse }}$ H69E mutants carrying NL4-3 Q7, L33/N37 demon-

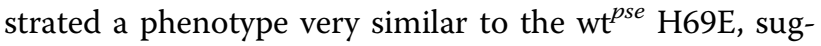
gesting that these residues contributed minimally to the rescue effect. In contrast, wt ${ }^{\text {pse }}$ H69E/A95C mutant, which contains single amino acid reversion at residue 95, showed a relative Gag processing activity close to NL4-3 H69E mutant, indicating that C95 could facilitate autoprocessing. Interestingly, the double mutation I63L/ A67C also demonstrated rescued Gag processing to a less extent (Figure 2 lane 6). To further pinpoint the contributing residue(s), we mutated each residue individually, and the resulting constructs showed that both rescued the activity similarly to the double mutation (Figure 2A). Based on these observations, we suggested that cysteine 95 is the primary residue facilitating
A

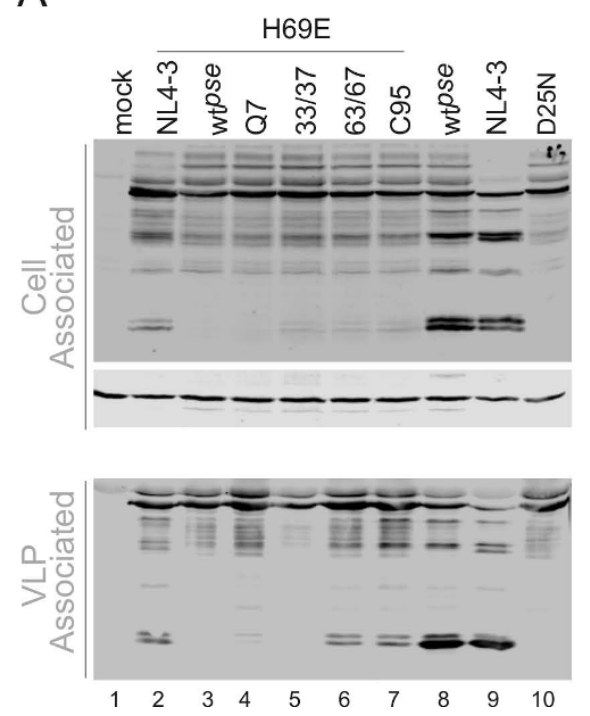

B

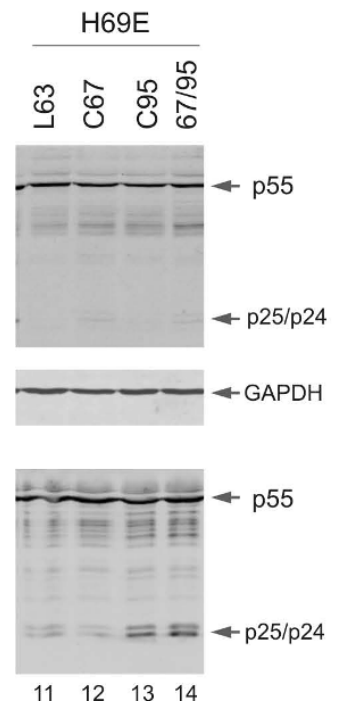

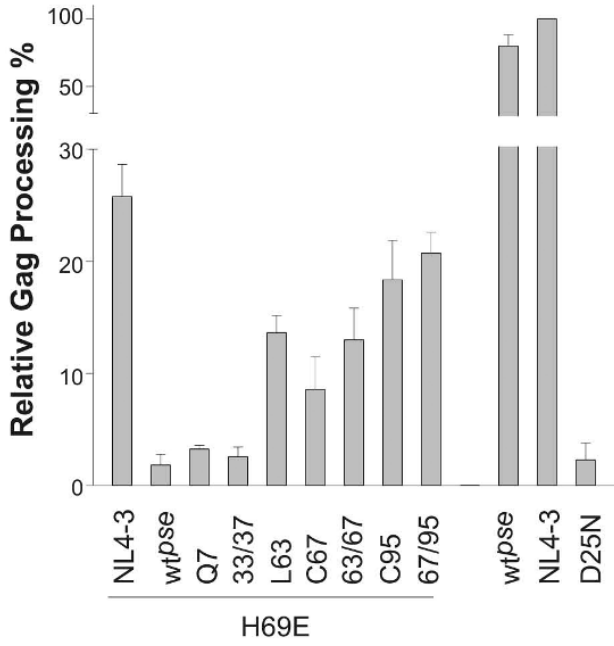

Figure 2 Cysteine 95 and other residues dampened the inhibitory effect of H69E on protease autoprocessing in transfected mammalian cells. (A) The indicated proviral DNAs were transfected into HEK 293T cells grown on 6-well plates with calcium phosphate. The total cell lysates and VLPs were prepared as described (Material and Methods) and subjected to western blot analysis. Mouse monoclonal antip24 antibody was used to detect proteins such as the full length Gag polyprotein (p55), CA-p2 intermediate (p25), and final processing product (p24) in the transfected cells and the released VLPs. The cell lysates blot was stripped and reprobed for GAPDH as loading controls. (B) Relative Gag processing efficiencies were quantified from three independent experiments and the bars represent standard deviations. 
protease autoprocessing and the subsequent Gag processing; L63 and C67 can also rescue the H69E inhibitory effect to a less extent probably because of the fact that they are in the close proximity to $\mathrm{H} 69$ residue in primary sequence. The double mutations, L63/C67 and C67/C95, only showed a slight enhancement on protease activity compared to the single mutations, indicating a lack of synergistic effect. We interpreted that these residues are capable of facilitating autoprocessing independently to a certain extent and these enhancements might be parallel to each other and not additive.

\section{H69D mutation abolishes protease autoprocessing even in the context of NL4-3 PR backbone}

In addition to H69E mutation, a previous study using bacterially expressed Gag-Pol precursor demonstrated inhibition of protease autoprocessing by H69D; whereas changes to $\mathrm{R}, \mathrm{L}, \mathrm{Y}, \mathrm{N}$, and $\mathrm{Q}$, individually, did not impair protease autoprocessing [29]. To compare H69E with H69D for their effects on protease maturation under the same context, we engineered a panel of mutations changing the parental $\mathrm{H} 69$ to D, N and Q individually in the pNL-PR backbone. As shown in Figure 3, VLPs produced by H69Q mutant displayed a p24 pattern similar to the wild-type control; and both H69N and $\mathrm{H} 69 \mathrm{E}$ showed partial Gag processing activities. In contrast, H69D VLPs only contained the full length p55 precursor; no processed intermediates or p24 were detected (Figure 3 lane 4), which resembled the D25N negative control. This data further verified that aspartic acid at position 69 significantly blocks protease maturation even in the presence of L63, C67, and C95. It is interesting that H69D mutation displays a more drastic inhibitory effect than H69E considering the carboxyl side chain of aspartic acid is only shorter by one methyl group $\left(-\mathrm{CH}_{2}\right)$ than that of glutamic acid. Quantitative analysis demonstrated relative Gag processing efficiencies following an order of wt $\cong \mathrm{H} 69 \mathrm{Q}>\mathrm{H} 69 \mathrm{~N}, \mathrm{H} 69 \mathrm{E}$ > H69D in VLPs produced from transfected mammalian cells (Figure 3B). By examining structures of these amino acids, it seemed that a combination of the carbonyl group and its close distance to the $\mathrm{C} \alpha$ plays a role in inhibiting protease maturation.

Steady state levels of mature protease detected in VLPs (Figure 3A, the bottom panel) also qualitatively correlated with the relative Gag processing activities (Figure 3B). A rabbit polyclonal anti-PR antibody detects both mature and precursor proteases, but the precursor band overlaps with a non-specific background band (Figure 3A lane 1), so we mainly focused on detection of mature protease. In VLPs produced by the wild type NL4-3 and wt ${ }^{p s e}$, mature protease is the primary product, consistent with the high Gag processing efficiencies. The $\mathrm{wt}^{p s e}$ mature protease appeared to be more than the NL4-3 mature protease probably due to its higher stability because of the mutations engineered to reduce autoproteolysis. In VLPs produced from H69Q, mature protease was the primary form similar to the

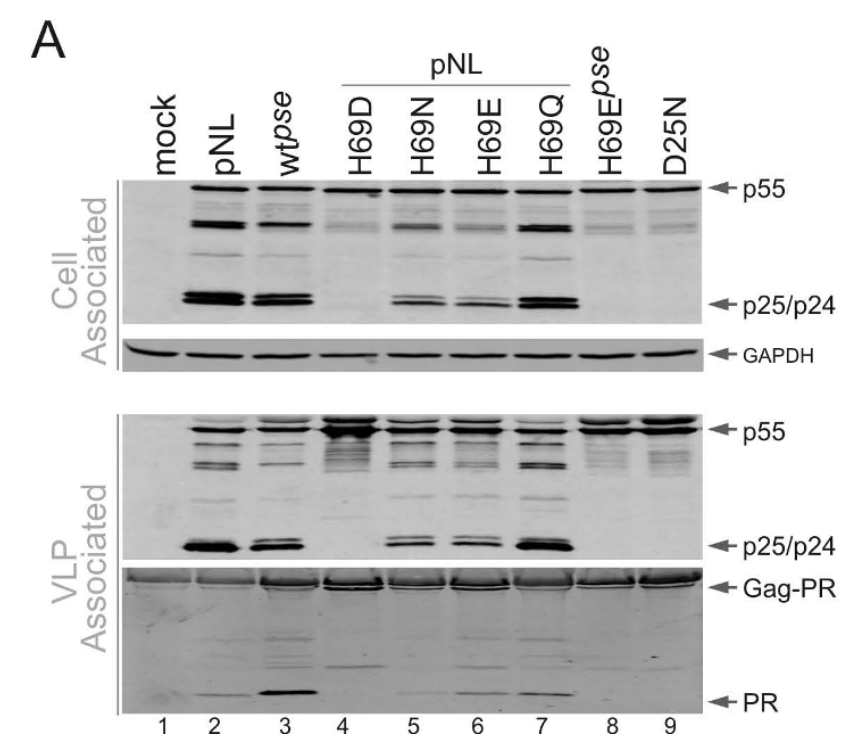

B

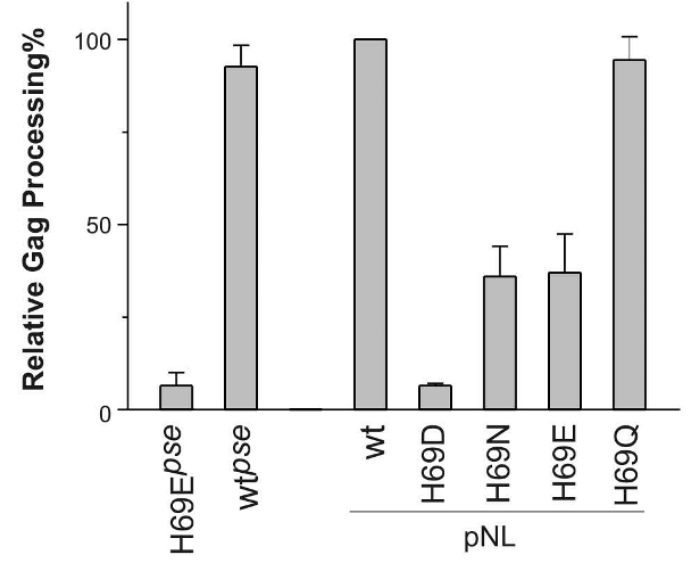

Figure 3 Different substitutions of $\mathbf{H 6 9}$ have differential effects on protease maturation. (A) HEK 293T cells grown on 6-well plates were transfected with the indicated proviral DNAs by calcium phosphate. The total cell lysates and VLPs were prepared as described (Material and Methods) and subjected to western blot analysis. Mouse monoclonal anti-p24 antibody was used to detect p24-containing proteins (p55, p25, and p24) in the transfected cells and the released VLPs. The cell lysates blot was stripped and reprobed for GAPDH as loading controls. VLP associated proteases were probed with polyclonal rabbit anti-PR antibodies. (B) Relative Gag processing efficiencies were quantified from three independent experiments and the bars represent standard deviations. 
wild type control. Consistent with the partial Gag processing activities, H69E and H69N VLPs contained reduced amounts of mature protease as well as partially processed intermediates. In D25N, H69D, and $\mathrm{wt}^{\text {pse }}$ H69E VLPs, minimal or no mature protease was detected; and the full length Gag-PR precursor appeared to be the predominant product.

\section{Charge substitutions of several residues did not rescue inhibition of $\mathrm{H} 69 \mathrm{E}$ on protease maturation}

Our mutagenesis analyses demonstrated that the negatively charged carbonyl group at close proximity to the $\mathrm{C}_{\alpha}$ of residue 69 inhibits protease maturation. Our previous study also suggested that H69E mutation inhibits in vitro autoprocessing probably by affecting proper precursor folding. One speculation is that positively charged side chains of the parental residues (H69 or K69) interact with another negatively charged residue to facilitate proper folding; and the carbonyl group of H69E disrupts the electrostatic interaction. To test this possibility, we performed a small scale screening for potential $\mathrm{H} 69$ interacting residues using a previously reported precursor autoprocessing assay [33]. When expressed in E. coli, GST-TFR-PR-FLAG fusion precursor autoprocesses releasing mature protease that can be detected in total lysates by Western blot (Figure 4 lane 1). H69E mutation significantly inhibits protease maturation (lane 3). We chose to mutate five surface residues (four acidic acids plus F99 that is in close proximity to H69) individually in the H69E context to examine whether a neutral or positively charged residue at these positions could rescue protease autoprocessing by complementing mutations. Out of a total of 12 constructs (E21K, E21Q, D31K, D31N, E34K, E35K, E34K/
E35K, F99K, F99N, F99Q, F99H, F99A), none of them reversed the inhibitory effect of $\mathrm{H} 69 \mathrm{E}$ on protease maturation (not all the mutants are shown here) and many of them further suppressed autoprocess activity (Figure 4, lanes 4-9). Consequently, our limited screening was unable to define residues that might interact with H69, and further examinations would be necessary to identify how $\mathrm{H} 69$ regulates protease maturation.

\section{Discussion and Conclusions}

Protease autoprocessing involves precursor dimerization and the $\mathrm{N}$-terminal cleavage that releases mature protease. In the infected cell, this process is also temporally correlated with the virion egress event. However, the molecular and cellular mechanisms underlying this highly regulated process are poorly understood. We previously reported that H69E mutation in a pseudo wild type protease sequence abolishes protease autoprocessing in E. coli and in transfected mammalian cells [33]. The current study demonstrates that L63, C67, and C95 dampen the H69E inhibitory effect. The Levine group also suggested a possible inter-play between H69 and C67 using a model peptide spanning residues 59 to 75 more than a decade ago [35]. It is interesting to note that highly conserved HIV-1 protease cysteines are not required for the catalytic activity, nor contributed to the formation of intramolecular disulfide bonds. Instead, they are thought to participate in redox regulation of protease activity [36,37] via a yet-to-be-defined mechanism. Both C67 and C95 appear to be sensitive to oxidation with C95 seems more accessible than C67 [36,38]. Glutathionylation of C67 increases and stabilizes protease activity in vitro, whereas C95 glutathionylation abolishes protease activity [38]. Using immature HIV
A

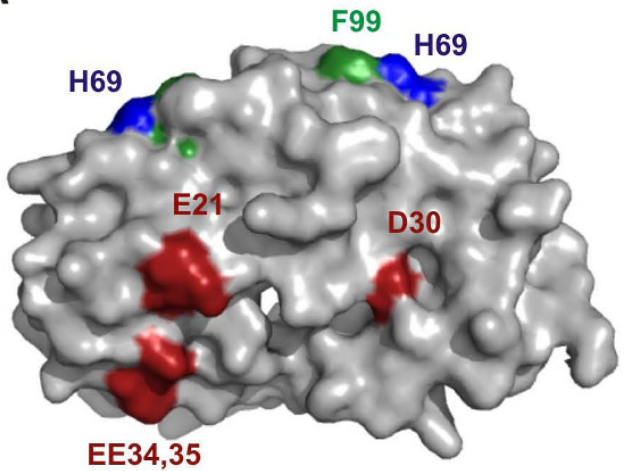

B

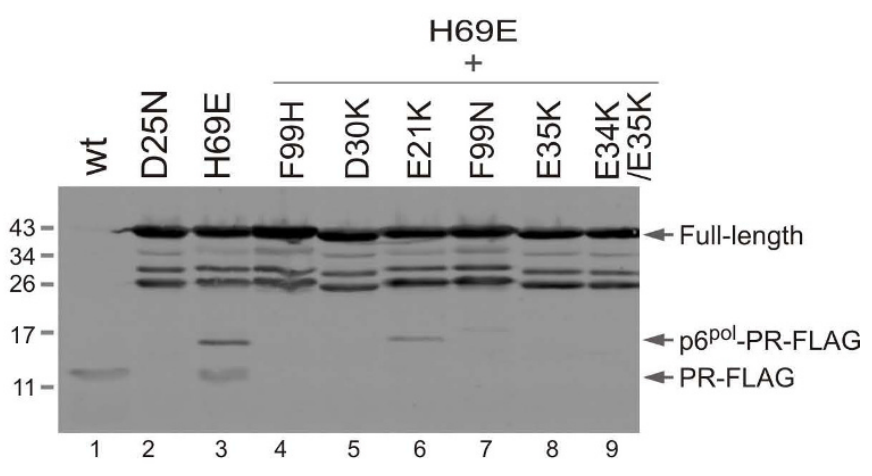

Figure 4 Charge substitutions of surface residues did not restore the inhibitory effect of H69E on protease autoprocessing. (A) Schematic presentation of the mature protease dimer (PDB 2PK6) with the surface residues that were tested in this report highlighted in red or green and histindine 69 in blue. (B) The pGEX-3X derived plasmids encoding for GST-TFR-PR-FLAG fusions bearing the indicated mutations were introduced into E. coli BL21(DE3) and induced for protein expression. The total lysates were prepared as described (Materials and Methods) and subjected to western blot analysis. A mouse anti-FLAG antibody was used to detect the full length precursor fusion, intermediates and mature protease (PR-FLAG). The denoted protein markers are in $\mathrm{kDa}$ for reference. 
virions produced in the presence of protease inhibitors as a model system, Davis et al. demonstrated that immature virions made from a mutant lacking the two cysteines undergo protease maturation at a higher rate than the wild type immature virions following the removal of inhibitors [37]. Reducing agent DTT enhances protease maturation, and oxidizing agents delay protease maturation of the immature virions. These results suggest an oxidation-and-reduction cycle that is involved in regulation of protease autoprocess. We envision that oxidation of cysteines prevents protease precursor from pre-maturation by locking it in an inactive status in the infected cell. Upon virion release, other factors trigger the reduction reaction that restores free cysteines rendering protease activity. This cysteine modification cycle seems unnecessary for protease autoprocessing and mature protease activity as the pseudo wild type protease containing mutations C67A/ C95A is able to process Gag polyprotein at levels comparable, yet slightly lower, to NL4-3 protease (Figure 2 and 3). However, in the context of H69E pseudo wild type protease, cysteine containing protease demonstrated a relative Gag processing activity higher than that lacking cysteines (Figure 2A). Therefore, the modification cycle might play an auxiliary role in concert with other regulation mechanisms to modulate protease autoprocessing.

Amino acid sequence alignment of HIV-1 proteases (HIV database - http://www.hiv.lanl.gov) indicates that residue 69 is mostly histidine or lysine and occasionally glutamine or tyrosine, which are neutral or positively charged. Previous studies [29,33] and current report also support the notion that a carbonyl group at close proximity to the $\mathrm{C} \alpha$ position of this residue inhibits protease autoprocessing. The H69 residue is exposed on the surface of mature protease dimer and is close to the C-terminus. It is intriguing that charge properties of a surface residue would have drastic effects on protease autoprocessing. Previous biochemical analyses demonstrated that H69E mutation significantly delays the TFR-PR precursor from autoprocessing in vitro; whereas the appropriately folded H69E mature protease only showed a slightly decreased catalytic activity [33]. This has led us to speculate that residue 69 is involved in autoprocessing by influencing precursor structure. We hypothesize that protease precursor undergoes conformational changes during autoprocessing and a carbonyl group close to the $\mathrm{C} \alpha$ of position 69 interferes with this pathway. It would be critical to identify residues that transiently interact with $\mathrm{H} 69$ during this process. Unfortunately, our limited screening was unable to define any of them. Extensive structural and biochemical analyses on the wild type and H69D precursor would be essential to provide insights into protease autoprocessing mechanisms.

\section{Methods \\ DNA mutagenesis}

Plasmids that were used in this report were generated with the standard molecular cloning procedures and the detailed sequence information is available upon request. Construction of pNL-PR was described previously [33], and all the pNL-PR mutants were derived from this vector by site-directed mutagenesis. Multiple D21, D30, E34, E35 and F99 substitutions were introduced into a pGEX-3X derived plasmid expressing GST-p6 $6^{\text {pol }}-\mathrm{PR}^{p s e}$ FLAG H69E was generated in a previous report [33]. All the plasmids were purified with QIAEX plasmid kits and verified by DNA sequencing.

\section{Cell culture, transfection and western blotting}

Human embryonic kidney derived 293T cells (ATCC, Manassas, VA) were maintained in DMEM with $10 \%$ fetal bovine serum and transfected by calcium phosphate as previously described [33]. In brief, 293T cells were plated in 6-well plates the night before to give 50$60 \%$ confluence at the time of transfection. One hour prior to the transfection, chloroquine was added to each well to a final concentration of $25 \mathrm{uM}$. A total of $1 \mu \mathrm{g}$ DNA in $131.4 \mu \mathrm{L}$ of $\mathrm{ddH}_{2} \mathrm{O}$ was mixed with $18.6 \mu \mathrm{l} 2$ $\mathrm{M} \mathrm{CaCl}_{2}$ to give a final volume of $150 \mu \mathrm{l}$. Then, $150 \mu \mathrm{l}$ of $2 \times \mathrm{HBS}$ was added dropwise to the DNA solution while mixing by vortex. The resulting mixture was directly added to the culture cells. After 7-11 h of incubation, the culture medium was replaced with chloroquine-free DMEM.

Total cell lysates were prepared as described previously $[33,39,40]$ to examine proteins in transfected cells. To examine proteins associated with the released virions, culture media collected from $11 \mathrm{~h}$ to $48 \mathrm{~h}$ post transfection was clarified of cell debris by a brief centrifugation $(20,800 \times \mathrm{g}$ for $2 \mathrm{~min}$ at ambient temperature) and the supernatant was transferred to another tube and centrifuged at $20,800 \times \mathrm{g}$ for $3 \mathrm{~h}$ at $4^{\circ} \mathrm{C}$ to pellet virions. Virion pellets were resuspended in $40 \mu \mathrm{l}$ of PBS for further analysis. About 1/6 of cell lysate made from each well was resolved through $10 \%$ SDS-PAGE and the proteins were transferred to a PVDF (Polyvinylidene Fluoride) membrane followed by western blot. Approximately one half of virus-like particles (VLPs) collected from each well were analyzed for p24 contents, and all the VLPs made from one well of a 6 -well plate were used for protease detection. Mouse anti-HIV p24 antibodies (Cat\# 3537) and rabbit anti HIV-1 protease serum (Cat\# 4105) were obtained from the NIH AIDS research and reference program. Mouse anti-GAPDH 
(clone 6C5) antibodies (Fisher Scientific, Pittsburgh, PA) were used to reflect cell numbers. IR800 labelled goat anti mouse or rabbit secondary antibodies were purchased from Rockland Immunochemicals Inc (Gilbertsville, PA) for western detection with an Odyssey infrared dual laser scanning unit.

\section{Quantification of relative Gag processing activity}

Western blot images that were captured by an Odyssey infrared dual laser scanning unit in tiff format were analyzed by Totallab software (Nonlinear Dynamics Inc., Newcastle upon Tyne, UK). Total pixel volume (less than the saturation threshold) of each band was quantified to represent band intensity that is assumed to be proportional to protein amounts as the blot was detected by monoclonal antibodies. The anti-p24 antibody is able to detect the full length (p55) Gag polyprotein as well as p25 (CA-p2), a processing intermediate, and p24, the final cleavage protein. Because the production of $\mathrm{p} 24$ from $\mathrm{p} 25$ is solely dependent on mature protease, the amounts of p24 in VLPs quantitatively correlate with the amounts of mature protease that indirectly reflect precursor maturation efficiencies. In this report, we calculated the ratio of $\mathrm{p} 24 /(\mathrm{p} 24+\mathrm{p} 25+\mathrm{p} 55)$ as a measure of Gag processing efficiency to indirectly represent autoprocessing activities with the value obtained from the wild type pNL-PR VLPs set as 100\% for normalization.

\section{Protease autoprocessing in $\mathrm{E}$. coli}

The pGEX-3X derived plasmids were transformed into BL21 cells (Novagen, San Diego, CA) and the individual colony was grown in $\mathrm{LB}$ medium at $37^{\circ} \mathrm{C}$ overnight. The overnight culture was then diluted 100 -fold into $2 \mathrm{xYT}$ and incubated at $37^{\circ} \mathrm{C}$ for another $2.5 \sim 3 \mathrm{~h}$ prior to the addition of IPTG $(40 \mu \mathrm{M})$ to induce protein expression. After IPTG induction at $30^{\circ} \mathrm{C}$ for $4 \mathrm{~h}$, cells $(\sim 30 \mu \mathrm{L})$ were directly mixed with $6 \times$ SDS loading buffer $(6 \mu \mathrm{L})$ and subsequently analyzed by $10 \%$ SDS-PAGE and Western blot. The full length GST-TFR-PR-FLAG precursor and mature protease (PR-FALG) along with processing intermediates were detected with mouse anti-FLAG antibody (Sigma, St. Luis, MO).

\footnotetext{
Acknowledgements

This work was supported in part by NIH, NIAID grant R21A1080351 to C. Chen. The following reagents were obtained through the AIDS Research and Reference Reagent Program, Division of AIDS, NIAID, NIH: HIV-1 p24 monoclonal antibody from Drs. Bruce Chesebro and Kathy Wehrly; HIV-1 protease antiserum from BioMolecular Technology (DAIDS, NIAID).
}

\section{Authors' contributions}

CC designed the project and wrote the manuscript. LH constructed the plasmids used in this study, performed 293T transfection and western blot analyses. AH carried out the E. coli protease maturation assay and participated in sequencing analysis of the constructs. All authors read and approved the final manuscript.

\section{Competing interests}

The authors declare that they have no competing interests.

Received: 10 November 2009 Accepted: 23 March 2010

Published: 23 March 2010

\section{References}

1. Barre-Sinoussi F, Chermann JC, Rey F, Nugeyre MT, Chamaret S, Gruest J, Dauguet C, Axler-Blin C, Vezinet-Brun F, Rouzioux C, Rozenbaum W, Montagnier $L$ : Isolation of a T-lymphotropic retrovirus from a patient at risk for acquired immune deficiency syndrome (AIDS). Science 1983, 220(4599):868-871.

2. Gallo RC, Salahuddin SZ, Popovic M, Shearer GM, Kaplan M, Haynes BF, Palker TJ, Redfield R, Oleske J, Safai B, et al: Frequent detection and isolation of cytopathic retroviruses (HTLV-III) from patients with AIDS and at risk for AIDS. Science 1984, 224(4648):500-503.

3. Scarlata S, Carter C: Role of HIV-1 Gag domains in viral assembly. Biochim Biophys Acta 2003, 1614(1):62-72.

4. Freed EO: HIV-1 gag proteins: diverse functions in the virus life cycle. Virology 1998, 251:1-15.

5. Louis JM, Weber IT, Tozser J, Clore GM, Gronenborn AM: HIV-1 protease: maturation, enzyme specificity, and drug resistance. Adv Pharmacol 2000, 49:111-146.

6. Chen C, Montelaro RC: Characterization of RNA elements that regulate gag-pol ribosomal frameshifting in equine infectious anemia virus. J Virol 2003, 77(19):10280-10287.

7. Oroszlan S, Luftig RB: Retroviral proteinases. Curr Top Microbiol Immunol 1990, 157:153-185.

8. Louis JM, Nashed NT, Parris KD, Kimmel AR, Jerina DM: Kinetics and mechanism of autoprocessing of human immunodeficiency virus type 1 protease from an analog of the Gag-Pol polyprotein. Proc Natl Acad Sci USA 1994, 91(17):7970-7974.

9. Pettit SC, Clemente JC, Jeung JA, Dunn BM, Kaplan AH: Ordered processing of the human immunodeficiency virus type $1 \mathrm{GagPol}$ precursor is influenced by the context of the embedded viral protease. $J$ Virol 2005, 79(16):10601-10607.

10. Ludwig C, Leiherer A, Wagner R: Importance of protease cleavage sites within and flanking human immunodeficiency virus type 1 transframe protein $\mathrm{p}^{*}$ for spatiotemporal regulation of protease activation. J Virol 2008, 82(9):4573-4584.

11. Louis JM, Ishima R, Torchia DA, Weber IT: HIV-1 protease: structure, dynamics, and inhibition. Adv Pharmacol 2007, 55:261-298

12. Tessmer U, Krausslich HG: Cleavage of human immunodeficiency virus type 1 proteinase from the $\mathrm{N}$-terminally adjacent $\mathrm{p}^{*}$ protein is essential for efficient Gag polyprotein processing and viral infectivity. J Virol 1998, 72(4):3459-3463.

13. Wondrak EM, Nashed NT, Haber MT, Jerina DM, Louis JM: A transient precursor of the HIV-1 protease. Isolation, characterization, and kinetics of maturation. J Biol Chem 1996, 271(8):4477-4481.

14. Cherry E, Liang C, Rong L, Quan Y, Inouye P, Li X, Morin N, Kotler M, Wainberg MA: Characterization of human immunodeficiency virus type-1 (HIV-1) particles that express protease-reverse transcriptase fusion proteins. J Mol Biol 1998, 284(1):43-56.

15. Paulus C, Ludwig C, Wagner R: Contribution of the Gag-Pol transframe domain $\mathrm{p}^{*}$ and its coding sequence to morphogenesis and replication of human immunodeficiency virus type 1. Virology 2004, 330(1):271-283.

16. Zybarth G, Carter C: Domains upstream of the protease (PR) in human immunodeficiency virus type $1 \mathrm{Gag}-\mathrm{Pol}$ influence PR autoprocessing. J Virol 1995, 69(6):3878-3884.

17. Pettit SC, Moody MD, Wehbie RS, Kaplan AH, Nantermet PV, Klein CA, Swanstrom R: The p2 domain of human immunodeficiency virus type 1 Gag regulates sequential proteolytic processing and is required to produce fully infectious virions. J Virol 1994, 68(12):8017-8027.

18. Pettit SC, Henderson GJ, Schiffer CA, Swanstrom R: Replacement of the P1 amino acid of human immunodeficiency virus type 1 Gag processing sites can inhibit or enhance the rate of cleavage by the viral protease. $J$ Virol 2002, 76(20):10226-10233.

19. Eizert H, Bander P, Bagossi P, Sperka T, Miklossy G, Boross P, Weber IT, Tozser J: Amino acid preferences of retroviral proteases for aminoterminal positions in a type 1 cleavage site. J Virol 2008, 82(20):10111-10117. 
20. Pettit SC, Lindquist JN, Kaplan AH, Swanstrom R: Processing sites in the human immunodeficiency virus type 1 (HIV-1) Gag-Pro-Pol precursor are cleaved by the viral protease at different rates. Retrovirology 2005, 2:66.

21. Liang C, Hu J, Russell RS, Roldan A, Kleiman L, Wainberg MA: Characterization of a putative alpha-helix across the capsid-SP1 boundary that is critical for the multimerization of human immunodeficiency virus type 1 gag. 2005, 76(22):11729-11737.

22. Kaplan AH, Zack JA, Knigge M, Paul DA, Kempf DJ, Norbeck DW, Swanstrom R: Partial inhibition of the human immunodeficiency virus type 1 protease results in aberrant virus assembly and the formation of noninfectious particles. J Virol 1993, 67(7):4050-4055.

23. Wiegers K, Rutter G, Kottler H, Tessmer U, Hohenberg H, Krausslich HG: Sequential steps in human immunodeficiency virus particle maturation revealed by alterations of individual Gag polyprotein cleavage sites. J Virol 1998, 72(4):2846-2854.

24. Li F, Goila-Gaur R, Salzwedel K, Kilgore NR, Reddick M, Matallana C, Castillo A, Zoumplis D, Martin DE, Orenstein JM, Allaway GP, Freed EO, Wild CT: PA-457: A potent HIV inhibitor that disrupts core condensation by targeting a late step in Gag processing. ProcNat/AcadSciUSA 2003, 100(23):13555-13560.

25. Koh Y, Matsumi S, Das D, Amano M, Davis DA, Li J, Leschenko S, Baldridge A, Shioda T, Yarchoan R, Ghosh AK, Mitsuya H: Potent Inhibition of HIV-1 Replication by Novel Non-peptidyl Small Molecule Inhibitors of Protease Dimerization. J Biol Chem 2007, 282(39):28709-28720.

26. Pearl LH, Taylor WR: A structural model for the retroviral proteases. Nature 1987, 329(6137):351-354.

27. Sayer JM, Liu F, Ishima R, Weber IT, Louis JM: Effect of the active site D25N mutation on the structure, stability, and ligand binding of the mature HIV-1 protease. J Biol Chem 2008, 283(19):13459-13470.

28. Kohl NE, Emini EA, Schleif WA, Davis L, Heimbach JC, Dixon RA, Scolnick EM, Sigal IS: Active human immunodeficiency virus protease is required for viral infectivity. Proc Natl Acad Sci USA 1988, 85(13):4686-4690.

29. Loeb DD, Swanstrom R, Everitt L, Manchester M, Stamper SE, Hutchison CA: Complete mutagenesis of the HIV-1 protease. Nature 1989, 340(6232):397-400.

30. Huang M, Orenstein JM, Martin MA, Freed EO: p6Gag is required for particle production from full-length human immunodeficiency virus type 1 molecular clones expressing protease. JVirol 1995, 69:6810-6818.

31. Louis JM, Wondrak EM, Kimmel AR, Wingfield PT, Nashed NT: Proteolytic processing of HIV-1 protease precursor, kinetics and mechanism. J Biol Chem 1999, 274(33):23437-23442.

32. Tang C, Louis JM, Aniana A, Suh JY, Clore GM: Visualizing transient events in amino-terminal autoprocessing of HIV-1 protease. Nature 2008, 455(7213):693-696

33. Huang L, Sayer JM, Swinford M, Louis JM, Chen C: Modulation of human immunodeficiency virus type 1 protease autoprocessing by charge properties of surface residue 69. J Virol 2009, 83(15):7789-7793.

34. Louis JM, Clore GM, Gronenborn AM: Autoprocessing of HIV-1 protease is tightly coupled to protein folding. Nat Struct Biol 1999, 6:868-875.

35. D'Ettorre C, Levine RL: Reactivity of cysteine- 67 of the human immunodeficiency virus-1 protease: studies on a peptide spanning residues 59 to 75. Arch Biochem Biophys 1994, 313(1):71-76.

36. Davis DA, Newcomb FM, Starke DW, Ott DE, Mieyal JJ, Yarchoan R: Thioltransferase (glutaredoxin) is detected within HIV-1 and can regulate the activity of glutathionylated HIV-1 protease in vitro. J Biol Chem 1997, 272(41):25935-25940.

37. Davis DA, Yusa K, Gillim LA, Newcomb FM, Mitsuya H, Yarchoan R: Conserved cysteines of the human immunodeficiency virus type 1 protease are involved in regulation of polyprotein processing and viral maturation of immature virions. J Virol 1999, 73:1156-1164.

38. Karlstrom AR, Shames BD, Levine RL: Reactivity of cysteine residues in the protease from human immunodeficiency virus: identification of a surface-exposed region which affects enzyme function. Arch Biochem Biophys 1993, 304(1):163-169.

39. Chen C, Li F, Montelaro RC: Functional roles of equine infectious anemia virus Gag p9 in viral budding and infection. J Virol 2001, 75(20):9762-9770.
40. Chen C, Vincent O, Jin J, Weisz OA, Montelaro RC: Functions of early (AP2) and late (AIP1/ALIX) endocytic proteins in equine infectious anemia virus budding. J Biol Chem 2005, 280(49):40474.

doi:10.1186/1742-4690-7-24

Cite this article as: Huang et al: Cysteine 95 and other residues influence the regulatory effects of Histidine 69 mutations on Human Immunodeficiency Virus Type 1 protease autoprocessing. Retrovirology $20107: 24$

\section{Submit your next manuscript to BioMed Central and take full advantage of:}

- Convenient online submission

- Thorough peer review

- No space constraints or color figure charges

- Immediate publication on acceptance

- Inclusion in PubMed, CAS, Scopus and Google Scholar

- Research which is freely available for redistribution

Submit your manuscript at www.biomedcentral.com/submit
Biomed Central 